Revista Médica Sinergia Vol.3 Num:12

Diciembre 2018 pp: 13 - 24

ISSN:2215-4523

e-ISSN:2215-5279

http://revistamedicasinergia.com

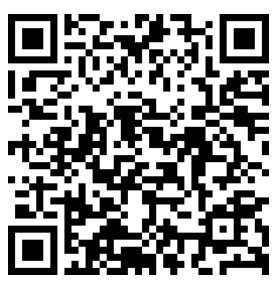

${ }^{1}$ Médico general, graduado de la Universidad de lberoamerica (UNIBE), médico en Clínica Carlos Durán Cartin, San José, Costa Rica.

Código médico: 15082 mgc11@hotmail.com

\section{NARCOLEPSIA: ABORDAJE DIAGNOSTICO Y TERAPEUTICO}

(Narcolepsy: diagnostic and therapeutic approach)

\author{
${ }^{1}$ Dr. Marco Tulio Gómez Cerdas \\ Clínica Carlos Durán Cartin, San José, Costa Rica \\ mgc11@hotmail.com \\ https://orcid.org/0000-0002-5329-3359
}

DOI: https://doi.org/10.31434/rms.v3i12.161

\title{
RESUMEN
}

La narcolepsia también conocida como síndrome de Gelineau o epilepsia del sueño. Es definida como una enfermedad neurológica caracterizada por somnolencia diurna excesiva, transiciones prematuras de la vigila al sueño con movimientos oculares rápidos (REM, Rapid Eyes Movements), parálisis al despertar o parálisis de sueño, alucinaciones hipnagógicas (visiones fugaces en la transición vigilia-sueño) o hipnopómpicas (transición sueño-vigilia), sueño nocturno fragmentado y en el caso de la narcolepsia tipo 1 sufren de cataplejía (períodos en los cuales el individuo presenta pérdida bilateral del tono muscular sin pérdida de la conciencia), hallazgo que no ocurre en la tipo 2. En esta revisión se realiza con el fin de promover el conocimiento de esta patología tanto para pacientes, familiares y profesionales de la salud para obtener un adecuado abordaje diagnóstico y terapéutico en esta población.

PALABRAS CLAVES: somnolencia, cataplejía, parálisis del sueño, narcolepsia, Síndrome de Gelineau.

\section{ABSTRACT}

Narcolepsy also known as Gelineau syndrome or sleep epilepsy. It is defined as a neurological disease characterized by excessive daytime sleepiness, premature transitions from sleep monitoring with rapid eye movements (REM), paralysis on awakening or sleep paralysis, hypnagogic hallucinations (fleeting visions in the wake-sleep transition) or hypnopompics (sleep-wake transition), fragmented nocturnal sleep and in the case of narcolepsy type 1 suffered from cataplexy (periods in which the individual presents bilateral loss of muscle tone without loss of consciousness), does not occur in type 2. This review is carried out in order to promote knowledge of this pathology for patients, family members and health professionals to obtain an adequate diagnostic and therapeutic approach in this population. 
KEY WORDS: somnolence, cataplexy, sleep paralysis, narcolepsy, gelineau syndrome.

\section{INTRODUCCIÓN}

El sueño es un fenómeno natural y un estado conductual reversible, que se acompaña de una desconexión del estado de percepción o del entorno, no obstante, es un estado dinámico donde grupos de neuronas siguen activas desempeñando un papel diferente al de la vigilia, el cual es necesario para consolidar las distintas formas de la memoria, almacenar energía, mantener la inmunocompetencia, regular la temperatura corporal y la función de ciertos neurotransmisores. ${ }^{4}$

Por otra parte, existen patologías que pueden llegar a afectar el ciclo del sueño, y una de ellas, es la narcolepsia, patología subdiagnosticada, considerada como un trastorno neurológico que provoca somnolencia diurna excesiva.

Esta enfermedad puede afectar las relaciones interpersonales, sociales, ocasionar pérdida de autoestima e inclusive puede ser causa de desempleo; la intención con este reporte, es promover el conocimiento de esta enfermedad, abordando desde su fisiopatología, sus criterios diagnósticos y el adecuado manejo terapéutico que rige actualmente.

\section{ANTECEDENTES}

La narcolepsia proviene del griego nárke, "sopor" y lepsis "posesión", conocida en sus inicios como síndrome de Gélineau o epilepsia del sueño, fue descrita en el año 1872 por Westphal. Sin embargo, fue tipificada por el neurólogo francés
Jean Baptiste Edouard Gélineau en 1880, describiendo a un paciente con somnolencia excesiva y episodios de debilidad muscular desencadenados por emociones, que denominó "astasia" o "caídas", seguidamente se conocerá con el término de "cataplejía" introducido por Robert Hennenerg, en 1916. 1,2

Las primeras observaciones de esta enfermedad fueron descritas como episodios de sueño irresistibles, de corta duración, con episodios de caídas e incluso la consideraron una neurosis rara o poco conocida. Posteriormente, Adie redefinió el concepto y la cataloga como entidad única, independiente de la neurosis y de otros trastornos de asociación frecuente como la epilepsia.

1,2

En 1916, Baron Constantin Von Economo, sugirió la participación del hipotálamo en la regulación del ciclo sueño-vigilia. Y durante la epidemia europea de encefalitis letárgica (19161927) observó que las lesiones en el hipotálamo posterior se asociaban con hipersomnia, mientras que las lesiones en el hipotálamo anterior ocasionaban insomnio. ${ }^{1}$

Daniels, en 1930 describió la "tétrada clínica", resaltando la asociación del sueño diurno excesivo o hipersomnia, cataplejía, parálisis de sueño y alucinaciones hipnagógicas. ${ }^{2}$

Para 1975, la palabra narcolepsia fue definida por los participantes del Primer Simposio Internacional sobre Narcolepsia, haciendo referencia a un síndrome de origen desconocido caracterizado por tendencias anormales 


\begin{tabular}{|c|c|c|}
\hline Insomnios & Trastornos respiratorios & Hipersomnias centrales \\
\hline $\begin{array}{l}\text { Insomnio crónico } \\
\text { Insomnio de corta evolución } \\
\text { Otros insomnios } \\
\text { Variantes } \\
\text { - Excesivo tiempo en } \\
\text { cama } \\
\text { - Dormidor corto }\end{array}$ & $\begin{array}{l}\text { Apneas obstructivas del } \\
\text { sueño } \\
\text { •Del adulto } \\
\text { •Del niño } \\
\text { Apneas centrales del sueño } \\
\text { Trastornos de hipoventilación } \\
\text { Hipoxemia del sueño } \\
\text { Síntomas aislados o variantes } \\
\text { • Ronquido } \\
\text { - Catatrenia }\end{array}$ & $\begin{array}{l}\text { Narcolepsia tipo } 1 \text { (NT1) } \\
\text { Narcolepsia tipo } 2 \text { (NT2) } \\
\text { Hipersomnia idiopática } \\
\text { Síndrome de Kleine-Levin } \\
\text { Síndrome de sueño insuficiente } \\
\text { Hipersomnias secundarias } \\
\text { Variantes: dormidor largo }\end{array}$ \\
\hline $\begin{array}{l}\text { Trastornos del ritmo } \\
\text { sueño-vigilia }\end{array}$ & Parasomnias & Movimientos anormales \\
\hline $\begin{array}{l}\text { Fase de sueño retrasada } \\
\text { Fase de sueño avanzada } \\
\text { Fase de sueño irregular } \\
\text { Ritmo no de } 24 \text { horas } \\
\text { Trabajo en turnos } \\
\text { Jet-lag } \\
\text { Otros trastornos del ritmo }\end{array}$ & $\begin{array}{l}\text { No relacionadas con REM } \\
\text { Relacionadas con REM } \\
\text { Otras parasomnias } \\
\text { Síntomas aislados o variantes }\end{array}$ & $\begin{array}{l}\text { Piernas inquietas } \\
\text { Movimientos periódicos de los } \\
\text { miembros } \\
\text { Calambres del sueño } \\
\text { Bruxismo } \\
\text { Movimientos rítmicos relacionados con } \\
\text { el sueño } \\
\text { Mioclonías benignas del lactante } \\
\text { Mioclonías propioespinales } \\
\text { Otros, secundarios y no especificados }\end{array}$ \\
\hline
\end{tabular}

Fuente. El sueño y su patología, ICSD: International Classification of Sleep Disorders. ${ }^{15}$

manifestaciones patológicas en una etapa especial del sueño: el sueño paradójico o REM (Rapid Eyes Movements), apareciendo una duración acortada con respecto a los sujetos normales. $^{2}$

En 1990, fue publicada la primera clasificación de trastornos del sueño en el International Classification of Sleep Disorders, (ICSD-1). Posteriormente, esta clasificación fue modificada en el año 2005 (ICSD-2), incluyéndose a la narcolepsia al grupo de las hipersomnias de origen central, y en ese momento se consideró a esta enfermedad un cambio muy sustancial; sin embargo, para febrero de 2014 la American Academy of Sleep.

Medicine (AASM) publicó la tercera versión de la clasificación de trastornos del sueño (ICSD-3) que mantiene a

grandes rasgos el esquema previo, pero con cambios significativos en algunos de los capítulos, como por ejemplo describiendo a la narcolepsia en dos tipos: ((NT1) y (NT2)); clasificación que se mantiene actualmente. ${ }^{15}$ (Ver TABLA 3.1) 
Además de la nueva clasificación, la AASM ha ido publicando diferentes versiones de manuales para el estadiaje del sueño y de las anomalías del sueño (apneas, hipopneas, síndrome de piernas inquietas, bruxismo, alteraciones de frecuencia cardiaca, entre otras).

\section{EPIDEMIOLOGÍA}

La narcolepsia, es una de las causas más comunes de somnolencia crónica, afectando aproximadamente 1 de cada 2000 a 4000 personas. ${ }^{2,13}$

Por lo general, este trastorno comienza entre los 10 y 20 años de edad (40-50\% de casos en la adolescencia, y en un 2$5 \%$ antes de la pubertad), sin embargo, una vez establecido persiste de por vida; la narcolepsia puede permanecer sin diagnosticarse hasta en la mitad de los casos, principalmente porque algunos médicos no están familiarizados con este trastorno o se ignora en los casos de narcolepsia leve, formas incompletas o cuando se asocia a otros trastornos del sueño (como el síndrome de apnea obstructiva del sueño), afortunadamente, el conocimiento sobre esta patología y otros trastornos del sueño están en aumento, y en los últimos años se han logrado grandes progresos en la comprensión de la narcolepsia. 5,12, 13

El riesgo de presentar narcolepsia en familiares de primer grado es de $2 \%$, lo que implica un valor de 10 a 40 veces mayor que la población general. ${ }^{1} \mathrm{La}$ incidencia en gemelos monocigóticos es de un $25-31 \%$, lo que sugiere que no es suficiente la carga genética, sino que son necesarios otros factores de índole ambiental. ${ }^{12}$

Uno de los estudios más importantes en relación con la prevalencia de la narcolepsia, es el Finnish Cohort Study, basado en una población de etnia caucásica en el que participaron 16,179 pacientes gemelos monocigotos y dicigóticos. Se les realizó un cuestionario "Ullanlinna Narcolepsy Scale", mediante seguimiento telefónico y, previo a la finalización, mediante polisomnografía, obteniendo resultados de prevalencia de $0.026 \%$ (con un intervalo de confianza de 95\%, 0.0-0.06). ${ }^{2}$

Estudios de prevalencia en otras poblaciones tales como Gran Bretaña, Francia, República Checa, han arrojado valores semejantes: 0.02 a 0.067. Esta prevalencia es muy similar a la esclerosis múltiple y algunos autores proponen que los bajos registros se deben al subdiagnóstico o el desconocimiento de la enfermedad. ${ }^{2}$

\section{ETIOLOGÍA Y FISIOPATOLOGÍA}

La causa de la narcolepsia es desconocida. Sin embargo, en 1998, dos grupos independientes descubrieron simultáneamente un nuevo sistema de neurotransmisión; Luis de Lecea y col., por un lado, identificaron 2 péptidos a los que denominó hipocretinas por su localización en el hipotálamo y por su similitud con la hormona secretina, Hcrt-1 y Hcrt-2, (estas son estimulados por un gen, localizado en el cromosoma 17q21221 que codifica la síntesis de preprohipocretina para la producción de estas); mientras que el segundo grupo por Takeshi Sakurai y col., mediante purificación y aislamiento de ligandos de receptores huérfanos acoplados a proteínas $\mathrm{G}$, aislaron dos receptores que se unían a dos moléculas, identificadas con orexina $A$ y $B,(O R X-A$ y ORX-B); del griego orexis $=$ apetito, por el conocido 
papel del hipotálamo lateral en la regulación del apetito y el incremento en el consumo de alimento luego de la inyección intraventricular de estos neuropéptidos. Poco tiempo después se confirmó que eran el mismo péptido. 1,9, 12 Se sabe que en el hipotálamo es una de las áreas primordiales en la regulación del sueño y la alerta.

Hay 4 núcleos hipotalámicos principales implicados estrechamente relacionados con la activación de la regulación de la vigilia y el sueño:

1. El núcleo hipotalámico posterior con las hipocretinas para la vigilia.

2. El núcleo ventral lateral preóptico para el sueño NOREM.

3. El núcleo reticularis pontis oralis lateral para el sueño REM, y

4. El núcleo supraquiasmático para la regulación (reloj o marcapaso) del ciclo sueño-vigilia. ${ }^{4,12}$

Estudios han demostrado que la narcolepsia es debido a la pérdida de las neuronas hipotalámicas que sintetizan los neuropéptidos orexina o también llamados hipocretinas. Aunque las mutaciones genéticas rara vez causan narcolepsia humana, los investigadores pronto descubrieron que los pacientes con este trastorno tienen concentraciones muy bajas 0 indetectables de orexinas en el líquido cefalorraquídeo, y los estudios con necropsias mostraron la pérdida casi completa de neuronas productoras de orexina en el hipotálamo. ${ }^{5}$

En condiciones normales, las orexinas inducen episodios prolongados de vigilia y suprimen el sueño, por lo que la pérdida de la señalización de orexina causa intrusiones frecuentes del sueño durante el periodo usual de vigilia, con sueño REM y fragmentos de sueño REM en cualquier momento del día. ${ }^{5}$

Se ha encontrado evidencia que sugiere la probabilidad de que un proceso autoinmunitario produzca esta pérdida selectiva de neuronas generadoras de orexina, y se han encontrado ciertos antígenos leucocitarios humanos (HLA, human leucocyte antigens), que pueden aumentar el riesgo de trastornos autoinmunitarios (descrita por primera vez en 1983). ${ }^{1,5}$

El HLA DQB1*06:02 se encuentra en casi $90 \%$ de las personas con narcolepsia, pero sólo en 12 a $25 \%$ de la población general. Los investigadores postulan la hipótesis de que en personas con DQB1*06:02, una respuesta inmunitaria contra influenza, estreptococo u otras infecciones también puede dañar las neuronas productoras de orexina a través de un proceso de mimetismo molecular. Este mecanismo podría explicar el aumento de ocho a 12 veces en los casos nuevos de narcolepsia entre los niños de Escandinavia y algunas otras partes de Europa que recibieron una marca particular de vacuna contra influenza AH1N1 (Pandemrix, producida en Alemania). 1,5,13

En esas regiones, el número de casos nuevos de narcolepsia aumentó, con la aparición de los síntomas 1 a 2 meses después de la vacunación, principalmente en niños y adolescentes portadores del gen DQB1 ${ }^{*}$ 06:02. ${ }^{13}$

En China, la infección por influenza H1N1 fue común en el invierno de 20092010, y los nuevos casos de narcolepsia se triplicaron en el año siguiente. Esta oleada en nuevos casos sugiere que la combinación de $\mathrm{DQB} 1{ }^{*} 06: 02$, la edad joven y los estímulos inmunes 
particulares

considerablemente el riesgo de narcolepsia. ${ }^{13}$

En un estudio realizado en Canadá, específicamente con población de Quebec, con la vacuna Arepanrix, se obtuvieron resultados consistentes con un riesgo de narcolepsia y cataplejía en una pequeña magnitud posterior a la administración de la vacuna contra la pandemia de $\mathrm{AH} 1 \mathrm{~N} 1$ (aproximadamente un caso por millón de dosis administradas, menor en comparación a la Pandemrix). Y se determinó que existe preferencia en las personas menores de 20 años, con un inicio de alrededor 16 semanas posterior a la administración de la vacuna. ${ }^{8,13}$

Todo esto sugiere que los dos posibles factores etiológicos principales son:

1. Alteración del sistema hipocretina/ orexina.

2. Alelo mutado HLA II en leucocitos (DQB1*0602).

Sin embargo, en raras ocasiones, la narcolepsia puede ocurrir en trastornos neurológicos como sarcoidosis, desmielinización, tumor o trastorno para neoplásico 0 accidentes vasculares cerebrales que causan daño directo a las neuronas productoras de orexina en el hipotálamo o sus proyecciones. ${ }^{13}$

\section{DIAGNOSTICO}

El diagnóstico de narcolepsia a menudo es evidente a partir de la historia clínica, pero es esencial confirmar el diagnóstico con un polisomnograma seguido de una prueba o test de latencia múltiple del sueño (TLMS). ${ }^{13}$

El TLMS, en la actualidad es la prueba complementaria básica para el diagnóstico, midiendo la tendencia al sueño y la aparición o no de sueño REM. Consiste en 5 siestas de 20 minutos cada una con intervalos de 2 horas, la prueba generalmente comienza a las 8 a.m. y termina aproximadamente a las 5 a 6 p.m. El resultado del test se considera como patológico si la latencia de sueño es menor o igual a 8 minutos y la presencia de dos o más entradas en sueño REM, en las personas sanas generalmente se quedan dormidas en 15 minutos o más. ${ }^{13,9}$

La polisomnografía (PSG), además de ser un requisito previo al TLMS se realiza para asegurar una buena eficiencia del sueño y permite descartar otros trastornos del sueño que causen excesiva somnolencia diurna, fundamentalmente el síndrome de apnea hipoapnea obstructiva del sueño. ${ }^{9}$

En la narcolepsia lo específico en la PSG es una latencia de REM menor de 20 minutos en el $40-50 \%$ de los pacientes y una latencia de sueño menor de 10 minutos.

Los pacientes no deben realizar el día anterior actividades de gran esfuerzo y se deben retirar los medicamentos estimulantes y supresores de REM tres semanas antes, ya que pueden llevar a falsos positivos tanto en la PSG como en el TLMS. ${ }^{5,9}$

Además, se debe alentar a los pacientes para que duerman el tiempo suficiente durante la semana previa a la prueba para eliminar cualquier efecto del sueño insuficiente. $^{5}$

Otra herramienta diagnostica es la medida en líquido cefalorraquídeo (LCR) el cual se miden los niveles de Hipocretina-1 (Hcrt 1), porque además de ser la más específica es la única que se puede determinar. Es una técnica no estandarizada de la que no se dispone 
en todos los centros. Se considera diagnóstica cuando es inferior a 110 $\mathrm{pg} / \mathrm{ml}$, siendo prácticamente inexistente en el LCR de pacientes narcolépticos. Los resultados entre 110 y $200 \mathrm{pg} / \mathrm{ml}$ son inciertos 0 indeterminados. Es muy específica en pacientes narcolépticos con cataplejía (99\%) pero la sensibilidad es baja (16\%) en casos sin cataplejía o con cataplejía atípica. ${ }^{9}$

Además, esta prueba puede estar alterada en otras patologías como el síndrome de Guillain Barre. No es sensible a fármacos psicotrópicos u otros trastornos del sueño comunes. ${ }^{9}$

Las principales indicaciones de esta prueba son: TLMS dudosos o en niños a quienes no se les puede realizar un TLMS, pacientes con diagnóstico reciente de narcolepsia con cataplejía con TLMS negativos, pacientes que no pueden suspender los tratamientos psicótropos y fallos en el tratamiento. ${ }^{9}$

La última herramienta diagnostica es la tipificación de HLA-DQ para DQB1 ${ }^{*} 0602$, estos alelos son positivos en el $90 \%$ de pacientes con narcolepsia con cataplejía, y en el $70 \%$ en narcolepsia familiar. Pero en narcolepsia sin cataplejía disminuye al $40 \%$ y se da en un $24 \%$ de pacientes normales por lo que su especificidad es muy baja, del $8 \%$ al $38 \%$. Esta técnica no está incluida en los criterios diagnósticos de narcolepsia. ${ }^{9}$

Con estas herramientas diagnosticas fue posible que se lograron establecer criterios diagnósticos para esta enfermedad y los dividiremos según los 2 tipos que rigen actualmente:

\section{NARCOLEPSIA TIPO 1}

Para su diagnóstico es necesaria la presencia de hipersomnolencia y los ataques de sueño durante al menos tres meses, y uno o dos de los siguientes criterios:

a) que el paciente sufra cataplejía con una duración de menos de 8 minutos $y$ al menos 2 entradas en REM en el test de latencias múltiples de sueño (TLMS);

b) una concentración de hipocretina-1 en LCR menor de $110 \mathrm{pg} / \mathrm{ml}$ o menor de un tercio del valor normal de ese laboratorio.

Existen dos subtipos relacionados con la NT1, la narcolepsia debida a un proceso médico (paraneoplásica anti-Ma2, tumor - lesión en hipotálamo, traumatismo craneoencefálico, etc.) y la narcolepsia sin cataplejía con bajos niveles en LCR de hipocretina $1 .{ }^{15}$

La cataplejía tiene mucha utilidad diagnóstica porque casi no ocurre en

otros trastornos. En contraste, las alucinaciones hipnagógicas (al inicio del sueño) o "hipnopómpicas" (al despertar del sueño) son ocasionales, y se describen como sensación enormemente vívidas y multi-sensoriales (lo que la diferencia de las alucinaciones esquizofrénicas), estas pueden ser auditivas, visuales, táctiles y consisten en sensaciones de „presencias extrañas" (sombras, bultos), ruidos en la habitación o sensación de levitar en el espacio. 5,10

Muchos trastornos pueden causar sensación dedebilidad, pero en la cataplejía, los pacientes describen una debilidad funcional definitiva (p. ej., habla farfullante "tartamudea, balbucea", la caída de una taza, tropezar con una silla) con desencadenantes emocionales consistentes como el júbilo al reír de un buen chiste, la sorpresa agradable del encuentro inesperado con un amigo o la ira intensa. ${ }^{5}$ 
Por último, les ofrezco esta nemotecnia que nos propone "Rafael Pelayo", (psiquiatría y profesor clínico de ciencias del comportamiento, del centro para la ciencia y medicina del sueño de Stanford), con la palabra CHESS (ajedrez, en inglés) para ayudarnos a recordar los 5 principales síntomas de la narcolepsia tipo 1:

Cataplejía, alucinaciones

Hipnagógicas, somnolencia diurna

Excesiva, parálisis del

Sueño, disrupción o fragmentación del Sueño.

\section{NARCOLEPSIA TIPO 2}

El nombre alternativo es "narcolepsia sin cataplejía". Para el diagnóstico requiere la clínica de hipersomnolencia durante 3 meses, y los siguientes criterios:

a) ausencia de cataplejía;

b) duración de menos de 8 minutos y al menos dos entradas en REM en el test de latencias;

c) hipocretinas no medidas 0 con valores superiores a $110 \mathrm{pg} / \mathrm{nl} \quad 0$ mayor de un tercio del valor medio;

d) y la ausencia de una explicación mejor por otros trastornos del sueño, medicaciones u otros procesos.

Si a lo largo de la evolución aparece cataplejía o se miden los niveles de hipocretina y están bajos, el diagnóstico sería de narcolepsia tipo 1 (suele ocurrir en un $10 \%$ de los casos). ${ }^{15}$

\section{TRATAMIENTO}

El tratamiento de la narcolepsia es sintomático. La mayoría de los pacientes con este trastorno se sienten más alertas después de dormir y se les debe alentar para dormir lo suficiente todas las noches y tomar una siesta de 15 a 20 min por la tarde; esta siesta puede ser suficiente para algunos pacientes con narcolepsia leve, pero la mayoría requiere tratamiento con fármacos que favorecen la vigilia. ${ }^{5}$

El modafinilo es el tratamiento de primera línea y tiene menos efectos colaterales en comparación con las anfetaminas, su semivida es relativamente prolongada; una dosis de 200 a 400 mg cada mañana es muy efectiva, su dosis máxima $600 \mathrm{mg}$ dividida en 2 tomas. ${ }^{5}$

Sus posibles mecanismos de acción son la estimulación adrenérgica alfa-1, interacciones en los sistemas dopaminérgicos o la participación de mecanismos

GABAérgicos (ácido gammaaminobutírico). Este fármaco puede aumentar la concentración hepática de las enzimas del citocromo P450 y puede aumentar el metabolismo de los anticonceptivos orales $u$ otros medicamentos que tengan participación con este citocromo, por esto se debe analizar las interacciones medicamentosas antes de iniciarlo. Sus principales efectos adversos suelen ser cefalea, náuseas $\mathrm{y}$, en ocasiones, rinitis. $^{3,9}$

Como medicamento de segunda línea está indicado el metilfenidato $(10-20 \mathrm{mg}$ c/12 h) a menudo es efectivo, y este actúa induciendo la liberación de dopamina, y produce como efectos secundarios: irritabilidad, hiperactividad, alteraciones del estado de ánimo, cefalea, palpitaciones, sudoración, temblores, anorexia, insomnio, ansiedad, además existe la posibilidad de abuso del fármaco por lo que lo hace preocupante. $^{9}$ 
Las anfetaminas son la tercera línea de tratamiento principalmente para la narcolepsia tipo 1 , su mecanismo de acción es aumentar la concentración de dopamina y norepinefrina. La única anfetamina aprobada es la dextroanfetamina, pero está aprobada solo en algunos países. Ladosis recomendada comienza con $5 \mathrm{mg}$ por día hasta un máximo de $60 \mathrm{mg}$ por día. ${ }^{3}$ Los efectos secundarios son bastante similares a los del metilfenidato (irritabilidad, agresividad, insomnio e hipertensión, en dosis altas, también pueden inducir movimientos anormales, arritmias cardíacas y síntomas psicóticos). Por esta razón, las anfetaminas no se recomiendan en pacientes con antecedentes de enfermedad cardiovascular. ${ }^{3}$

A pesar de la posibilidad de abuso de drogas 0 tolerancia al usar psicoestimulantes, los pacientes narcolépticos rara vez presentan adicción a este medicamento. ${ }^{3}$

Entre otras opciones terapéuticas se encuentra el oxibato de sodio (hidroxibutirato $\mathrm{y}$ ) se administra a dosis inicial de $4,5 \mathrm{~g} / \mathrm{noche}$ dividida en 2 tomas iguales de 2,25 g, hasta un máximo de 9 $\mathrm{g} /$ noche con incrementos progresivos de $1,5 \mathrm{~g}$, a menudo es muy valioso para mejorar el estado de alerta, también es muy efectivo para reducir la cataplejía. Este actúa como un neurotransmisor/ neuromodulador natural actuando a través de sus propios receptores o a través de la estimulación de los receptores GABA-B24. ${ }^{5,9}$

Los efectos secundarios descritos principalmente son las náuseas, sedación excesiva, enuresis, despertares con confusión y cefalea; generalmente se usa solo en personas con somnolencia moderada a severa 0 cataplejía. Y no se recomienda durante el embarazo. ${ }^{13,9}$

Para los pacientes con cataplejía se ha visto que mejoran mucho con antidepresivos, por ejemplo, los que aumentan el tono noradrenérgico 0 serotoninérgico ya que estos fármacos suprimen el sueño REM y la cataplejía. Entre estos medicamentos tenemos a la venlafaxina (37.5-150 mg cada mañana) y la fluoxetina (10-40 mg cada mañana) a menudo son muy efectivas. ${ }^{5,9}$

Los antidepresivos tricíclicos, como la protriptilina (10-40 $\mathrm{mg} / \mathrm{día}) \quad 0 \quad$ la clomipramina (25-50 mg/día) son supresores potentes de la cataplejía, pero sus efectos anticolinérgicos, incluida la sedación, xerostomía, sudoración, estreñimiento, taquicardia, aumento de peso, hipotensión, dificultad miccional e impotencia los hacen menos atractivos. ${ }^{5,9}$

Es importante que las personas con narcolepsia tomen en cuenta el riesgo de accidentes automovilísticos (aumenta en un factor de tres a cinco); por lo tanto, algunas personas pueden optar por tomar un estimulante antes de conducir, manejar solo por períodos cortos o no conducir el cual es el más recomendado. ${ }^{13}$

Con respecto al trabajo, las personas con narcolepsia pueden prosperar en entornos estimulantes como la enseñanza, pero en trabajos que puedan cursar sedentarios o que requieren una atención sostenida pueden ser una mala opción. ${ }^{13}$

La cafeína, un antagonista del receptor de adenosina $\mathrm{A} 1$ y $\mathrm{A} 2 \mathrm{a}$, y las bebidas energéticas son a menudo estimulantes para combatir el sueño excesivo, pero presenta una eficacia limitada y produce 
algunos efectos secundarios que pueden ser perjudiciales. La educación siempre es necesaria para ayudar a los pacientes a reconocer y actuar contra los síntomas, y para definir, junto con su médico, los objetivos del tratamiento. ${ }^{3}$

\section{FUTURO DEL TRATAMIENTO DE LA NARCOLEPSIA}

Existen tres tratamientos emergentes principales, los cuales se encuentran en estudio:

1. Tratamientos sintomáticos de modulación endocrina/transmisora: Agonistas selectivos de la histamina (antagonistas $\mathrm{H} 3$ ), antagonistas de la hormona liberadora del crecimiento $(\mathrm{GHRH})$, agonistas del gammahidroxibutirato (GHB) y agonistas del ácido gamma-aminobutírico tipo $B$ (GABA-B). ${ }^{9}$

Con el uso de los antagonistas del receptor histaminérgico de tipo 3 se evidenció una reducción de la somnolencia diurna en las evaluaciones subjetivas y objetivas, pero, los síntomas específicos, como la cataplejía, las alucinaciones y la parálisis al despertar no experimentaron cambio alguno. ${ }^{12}$

El principal selectivo agonista inverso del receptor H3 de histamina, "Pitolisant", actúa de forma presináptica y activa las neuronas de histamina, Recientemente ha sido aprobado por la European Medicine Agency (EMA) y está disponible en Europa, pero no en los EE. UU. La dosis diaria inicial es de $10 \mathrm{mg}$ y puede aumentarse hasta $40 \mathrm{mg}$, administrada una vez al día. Generalmente es bien tolerado, con solo unos pocos eventos adversos que incluyen dolor de cabeza, náuseas e insomnio. ${ }^{3}$

2. Tratamientos basados en la hipocretina: Agonistas de la hipocretina y trasplantes celulares, orientados hacia la reposición de hipocretina 0 incluso prevenir la pérdida de neuronas que contienen este neuropéptido. ${ }^{9}$

3. Tratamientos de base inmunitaria. Corticoides, Ig IV y la plasmaféresis. ${ }^{9}$ Con la hipótesis autoinmunitaria se ha sugerido que un tratamiento inmunosupresor de forma muy precoz podría parar la progresión de la pérdida de neuronas hipocretinérgicas, principalmente cuando ésta puede ser irreversible; aunque la experiencia del tratamiento con inmunoglobulinas en dichos casos ha tenido resultado muy variables, con una mejoría subjetiva, no se ha visto tan evidente con pruebas objetivas, como el test de latencias múltiples del sueño o los valores normales de Hcrt-1 en el líquido cefalorraquídeo. ${ }^{12}$

\section{CONCLUSIONES}

La narcolepsia es una enfermedad infradiagnosticada en la población general, puede deberse a la baja incidencia documentada; la intención con este reporte es que tanto médicos de atención primaria como médicos especializados puedan abrir el conocimiento sobre esta patología, y así lograr un adecuado abordaje clínico.

Aunque es una patología compleja y de etiología indeterminada, los criterios diagnósticos de la narcolepsia, ya sea tipo 1 o tipo 2, permitirán identificar adecuadamente a esta población, y se espera en un futuro no muy lejano, poder 
determinar si estamos en frente a una patología de carácter inmunológico o de tipo destructivo (teoría hipocretina/ orexina).

Por el momento, nos apoyaremos con los estudios de la polisomnografía y el test de latencias múltiples del sueño como complemento para el diagnóstico, y aunque no existe una cura definitiva, se mantendrán estos pacientes bajo tratamiento sintomático, dirigido e individualizado, priorizando siempre sus riesgos, como lo son al conducir, operar maquinarias 0 en actividades que ameriten de sumo cuidado.

\section{BIBLIOGRAFÍA}

1. Bermúdez, J. (2014) Enfermedad laboral relacionada a trastornos del sueño por alteración del ritmo circadiano (TSRC). A propósito de un caso. Medicina Legal de Costa Rica - Edición Virtual. 31, 3-7.

2. Arias, O. (2009) Sistema hipocretinérgico y narcolepsia. Revista Médica de Chile. 137, 12091216.

3. Baccelli, A.; Pedemonte, M. (2013) Narcolepsia, trastorno del sueño subdiagnosticado. Tendencias en Medicina. 42: 96-103.

4. Urrestarazu, E.; Escobar, F. \& Iriarte, J. (2015) El sueño y su patología. Medicine - Programa de Formación Médica Continuada Acreditado.11, 4385-4394.

5. Scammell, T. (2015) Narcolepsy. The New England Journal Medicine. 373, 2654-2661.

6. Kasper, D.; Fauci, A.; Hauser, S; Longo, D.; Jameson, J. \& Loscalzo, J. (2016). Harrison. Principios de Medicina Interna, 19e ed. McGraw-Hill. 184-190.

7. Santamaría, J. (2012) Actualización diagnóstica y terapéutica en narcolepsia. Revista de Neurología; 54, 25-30.

8. Pabón, R.; García I.; Morales, G.; Urriza, J.; Imirizaldu, L. \& Ramos, F. (2010) Narcolepsia: actualización en etiología, manifestaciones clínicas y tratamiento. Anales del Sistema Sanitario de Navarra. Vol. 33, № 2, 191-200.

9. Montplaisir, J.; Petit, D.; Quinn, M.; Ouakki, M.; Deceuninck, G.; Desautels, A.; Mignot, E. \& De Wals, P. (2014) Risk of Narcolepsy Associated with Inactivated Adjuvanted (AS03) A/H1N1 (2009) Pandemic Influenza Vaccine in Quebec. PLOS ONE. 9, 1-8.

10. Peraita, R. (2015). Narcolepsia con cataplejía (narcolepsia tipo 1). D"genes, Asociación de enfermedades raras. 1-2. Accedido y descargado el 20 de octubre 2018, desde https://www.narcolepsia.org/app/download/24762736/VIII+Congreso+Nacional+de+Enfermeda des+Raras.pdf 
11. Barateau, L., Lopez, R., \& Dauvilliers, Y. (2016). Treatment Options for Narcolepsy. Springer International Publishing Switzerland. 1-8.

12. Lammers, G. (2018) Drugs Used in Narcolepsy and Other Hypersomnias. Sleep Medicine Clinics. 13, 183-189.

13. Merino, A., Hidalgo, V. (2010) Hipersomnia. Somnolencia diurna excesiva y alteraciones del ritmo circadiano en pediatría. Pediatría Integral. XIV (9), 722-724.

14. Romigi, A.; Vitrani, G.; Lo Giudice, T.; Centonze, D.; Franco, V. (2018) Profile of pitolisant in the management of narcolepsy: design, development, and place in therapy. Drug Design, Development and Therapy.12, 2665-2675.

15. Song, H.; Kim, T.; Um, Y. \& Hong, S. (2016). Narcolepsy: Association with H1N1 Infection and Vaccination. Sleep Medicine Research. 7(2):43-47.

16. Zhang, J.; Han, F. (2017) Sleepiness in Narcolepsy. Sleep Medicine Clinics. 1-7.

Recepción: 20 Octubre de 2018

Aprobación: 15 Noviembre de 2018

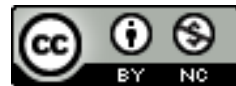

Reconocimiento-No Comercial 4.0 Internacional (CC BY-NC 4.0)- (BY) Debe reconocer adecuadamente la autoría, proporcionar un enlace a la licencia e indicar si se han realizado cambios<. Puede hacerlo de cualquier manera razonable, pero no de una manera que sugiera que tiene el apoyo del licenciador o lo recibe por el uso que hace. (NC) No puede utilizar el material para una finalidad comercial. 